\title{
Kernos
}

Revue internationale et pluridisciplinaire de religion grecque antique

6 | 1993

Varia

\section{L'Athéna de Platon}

\section{Catherine Lecomte}

URL : http://journals.openedition.org/kernos/548

DOI : $10.4000 /$ kernos.548

ISSN : 2034-7871

\section{Éditeur}

Centre international d'étude de la religion grecque antique

\section{Édition imprimée}

Date de publication : 1 janvier 1993

Pagination : 225-243

ISSN : 0776-3824

Référence électronique

Catherine Lecomte, "L'Athéna de Platon », Kernos [En ligne], 6 | 1993, mis en ligne le 07 avril 2011,

consulté le 01 mai 2019. URL : http://journals.openedition.org/kernos/548; DOI : 10.4000/kernos.548 


\section{L'ATHÉNA DE PLATON}

Les dieux des dialogues platoniciens sont-ils les mêmes que ceux d'Athènes? Et parmi eux, Athéna, principale déesse de la Cité, joue-t-elle un rôle prépondérant?

Le présent article voudrait, à partir d'un relevé des passages où Athéna est mentionnée, dégager le portrait sous lequel elle apparait, découvrir les traits de sa personnalité qui y sont mis en évidence ou qui, au contraire, y sont masqués, déceler une éventuelle exploitation philosophique de la figure divine.

Avant d'étudier les passages, les plus nombreux, où Athéna est associée à d'autres dieux, nous passerons en revue ceux dont elle est l'unique protagoniste. Et comment mieux aborder cette découverte de l'Athéna de Platon sinon par les étymologies de son nom proposées dans le Cratyle?

Lorsque Hermogène demande à Socrate d'expliquer les noms d'Athéna, d'Héphaistos et d'Arès' ${ }^{1}$, le philosophe commence par «l'autre nom de la déesse», "Pallas», car il n'est pas difficile à comprendre ${ }^{2}$ :

Eh bien donc, en pensant qu'il tire son origine de la danse en armes, nous aurions raison, je pense. Car le fait de s'élever en l'air, soi-même ou quelque chose d'autre, soit à partir de la terre soit avec les mains, c'est ce que nous

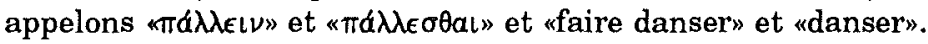

Au verbe verbe «mád $\lambda \epsilon \epsilon \nu$ » qui signifie «agiter vivement», Socrate ajoute l'idée de «danser en armes» 3 . Ce thème est développé dans un passage des Lois où il est rappelé comment Athéna,

1 Crat., 406d.

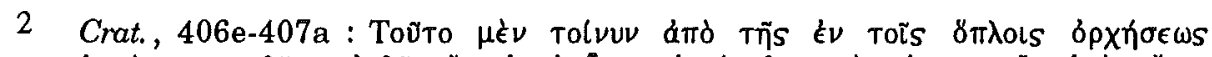

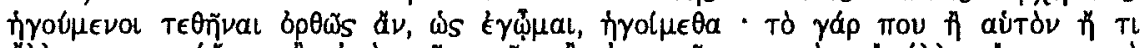

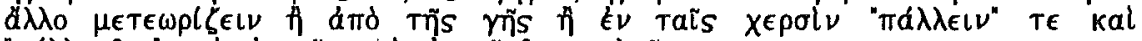

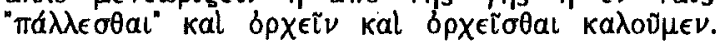

3 Le sens du mot «Pallas» reste obscur. Peut-être signifie-t-il «jeune-fille», cf. P. Chantraine, Dictionnaire étymologique de la langue grecque, Paris, 1968, p. 853-854. 
charmée par le jeu de la danse, pensa qu'elle ne devait pas se divertir les mains vides, mais que c'est entièrement parée de sa panoplie qu'elle devait accomplir cette danse 4 .

Quelques auteurs anciens ${ }^{5}$ montrent Athéna exécutant une danse armée ou lui attribuent l'invention de la pyrrhique, danse armée exécutée généralement avant ou après la bataille et qui donnait lieu à des concours lors des Panathénées. L'Athénien des Lois compare cette danse à celle des Courètes en Crète et des Dioscures à Lacédémone, et conseille aux jeunes gens de l'imiter afin de se préparer à la guerre et à la célébration des fêtes. La danse dont Athéna est l'inspiratrice fait donc partie du programme éducatif destiné aux enfants de l'État idéal. C'est un art qu'ils apprendront pour le pratiquer ensemble dans les moments importants de la vie de la communauté, la guerre et les fêtes.

Selon le Cratyle, le nom même de la déesse, "Athéna", révèle un autre aspect de sa personnalité. Toutes les étymologies proposées se rejoignent : Athéna est, pour les anciens et pour Homère, l'«intelligence» (voṽs) et la "réflexion" ( $\delta$ távota). Pour le "créateur des

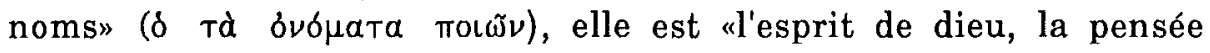
divine» ( $\theta \epsilon \circ \tilde{v}$ vónolv, \& $\theta \epsilon \circ \nu \delta \alpha$ ), "celle qui perçoit les choses divines

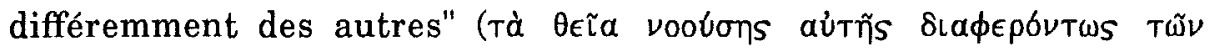

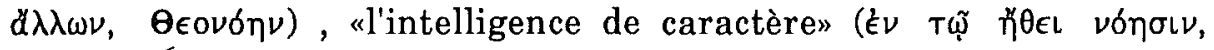
'Hoovónv) ${ }^{6}$.

Athéna, née de la tête de Zeus et fille de Mètis, personnifierait donc l'intelligence parmi les dieux. Pour Socrate, c'est d'après leur nature ( $\phi \dot{v} \sigma \epsilon l)$ que les choses sont nommées?. Le nom "Athéna» devrait donc révéler l'essence même de la déesse. Allons voir si les interventions de la déesse dans l'œuvre de Platon confirment cette qualité.

Le nom d'Athéna apparaît une nouvelle fois dans le Cratyle, lorsque Hermogène compare les explications complexes données par Socrate au

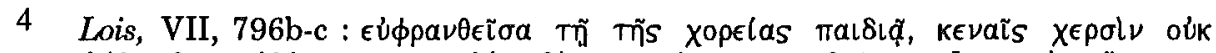

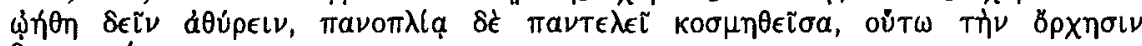
Slatepalvelv.

5 Denys D'Halicarnasse, Ant. Rom., VII, 72, 7; Lucien, Dialogues des dieux, 8 (226); ATHÉnÉE, IV, 184. Sur la pyrrhique, voir L. SÉCHAN, La danse grecque antique, Paris, 1930, p. $92-99$ et F. VIAN, La guerre des géants, Paris, 1952, p. 249-250.

6 Crat., 407a-c.

7 Crat., 383a-b, 390d-e, 391a. 
"prélude joué à la flûte du nome d'Athéna" ${ }^{8}$. Le vónos était un air liturgique exécuté en l'honneur d'un dieu par un chanteur, éventuellement accompagné d'un chœur; il comprenait un prélude et sept parties. C'était donc un chant assez complexe ${ }^{9}$ et on choisissait pour l'interpréter les meilleurs compositeurs et musiciens. Le nome était exécuté notamment lors des quatre grands Jeux ${ }^{10}$.

La mention du nome d'Athéna est assez rare : c'est Apollon le dieu musicien par excellence et c'est pour lui que le nome est généralement joué et chanté. Cependant, Athéna peut être également associée à la musique. C'est à elle que Pindare attribue l'invention du «nome à plusieurs têtes» ${ }^{11}$, et Pollux mentionne son nome ${ }^{12}$.

On voit que, de façon plaisante, Hermogène fait remarquer à Socrate qu'il se sent profane dans la science des noms, mais qu'avec l'aide du philosophe, il pourrait en percer les secrets et devenir tel un musicien qui maitrise son art au point de pouvoir interpréter un nome le nome d'Athéna plutôt que celui d'un autre dieu, car il est Athénien et discute avec un concitoyen.

Dans l'Hippias Majeur, Socrate se réfêre aussi à Athéna à propos de la définition du beau. Cherchant un exemple propre à prouver de façon indiscutable que le «beau» n'est pas l'«or», contrairement à ce que propose le sophiste, Socrate choisit la statue chryséléphantine d'Athéna qu'avait sculptée Phidias. Il sait en effet qu'Hippias reconnaitra sans discuter la beauté de cette œuvre et que la recherche d'une meilleure définition du beau pourra reprendre aussitôt. Et c'est effectivement ce qui se passe ${ }^{13}$.

Le passage apprend peu sur l'Athéna de Platon, il donnerait plutôt des indications sur l'Athènes du philosophe et du sophiste d'Élis. À moins que la déesse, comme sa statue, ne présente un harmonieux mélange de qualités qui la fasse briller d'un éclat particulier aux yeux de Socrate; à moins qu'Athéna, comme Phidias, ne soit passée maître

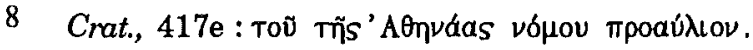

9 Sur la complexité du nome d'Athéna, cf. PLUT., Sur la musique, 1143b-c.

10 Cf. S. Michaelides, The Music of Ancient Greece. An Encyclopaedia, Londres, 1978, p. 222-223.

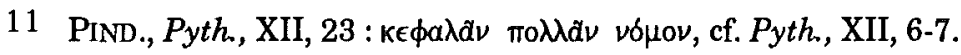

12 Pollux, IV, 57 et 66 . Cf. Michaelides, op. cit. (n. 10), p. 42 : Athéna aurait inventé l'aùós.

13 Hippias Majeur, 290 a-c. 
dans l'art de tirer le meilleur parti des «matières" mises à sa disposition...

La déesse est de nouveau prise comme modèle dans le Second Alcibiade. Lorsque le jeune homme réalise son besoin d'un professeur pour apprendre comment se comporter envers les dieux et les hommes, Socrate se propose d'être ce maître et se compare à Athéna ${ }^{14}$ :

Mais il me semble que, de même qu'Athéna, au dire d'Homère, chassa pour Diomède les ténèbres qui se trouvaient devant ses yeux «afin qu'il reconnût bien s'il avait affaire à un dieu ou à un homme», de même, pour toi aussi, il faut d'abord chasser les ténèbres de ton âme (...).

Athéna aime prendre un héros sous sa protection, de façon presque maternelle ${ }^{15}$. Elle guide à travers les ténèbres de l'ignorance un personnage, privilégié mais remarquable, qui ressent le besoin de cette aide et l'accepte. La collaboration entre la déesse et un mortel permet à chacun d'eux d'atteindre le but qu'il poursuit : à Athéna d'intervenir en faveur des Grecs pendant la guerre, à Diomède de s'illustrer dans la bataille; à Socrate de mener un jeune vers la philosophie, à Alcibiade de progresser dans la voie de la sagesse. Cette utilisation des qualités de chacun pour arriver au meilleur résultat est une des qualités d'Athéna qui apparait le plus souvent chez Platon.

Il est un dernier passage où Athéna n'est pas associée à d'autres dieux. Au début des Lois, l'Athénien interroge ses interlocuteurs, le Crétois Clinias et le Lacédémonien Mégillos, sur la guerre : si toute cité considère comme ennemies les autres cités, n'est-il pas nécéssaire qu'il en aille de même dans les rapports entre villages, entre individus, et même, que chaque homme se regarde comme son propre ennemi ? Et Clinias d'accueillir avec enthousiasme cette remarque :

Étranger athénien, je ne consentirais pas à t'appeler attique, car tu me sembles digne d'être appelé de préférence du nom de la déesse. Tu as rendu, en effet,

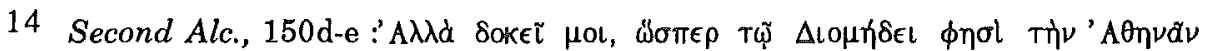

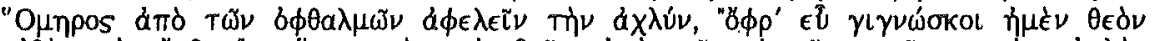

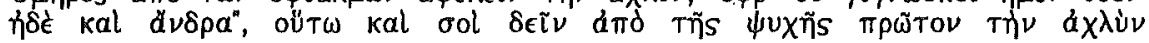

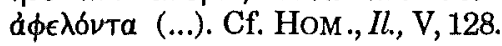

15 Cf. W. Burkert, Greek Religion. Archaic and Classical, trad. J. Raffan, Harvard, 1985, p. 141-142. 
notre raisonnement plus clair en le ramenant correctement à son principe (...) $)^{16}$.

Les interlocuteurs commencent à peine leur conversation lorsque Clinias fait ainsi l'éloge de l'Athénien. Mais il a suffi à ce dernier de quelques phrases pour définir au mieux le sujet du débat, pour montrer qu'il est un digne disciple de la déesse. Car il partage avec elle cette sagesse, cette intelligence "qui lui permet de comprendre les choses mieux que les autres» 17 . De même que Socrate se comparait à Athéna guidant Diomède dans la bataille, de même l'Athénien mérite-t-il de porter le nom de la déesse car il a engagé le débat sur la voie qui le mènera le plus sûrement à une conclusion fructueuse.

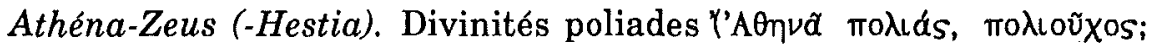

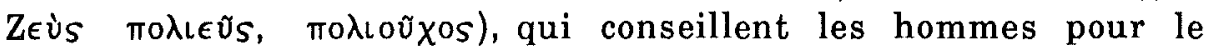

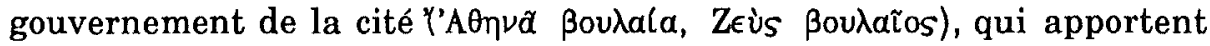
la victoire Y'A parfaitement. C'est avec son père qu'Athéna est le plus souvent associée par Platon. Ensemble, ils protègent la cité et garantissent le respect des lois 18 :

Mais si une personne qui a passé commande ne paie pas à l'artisan son salaire exactement selon l'accord convenu conformément à la loi, et ce au mépris de Zeus protecteur de la ville (moגıoṽxos) et d'Athéna qui participent tous deux au

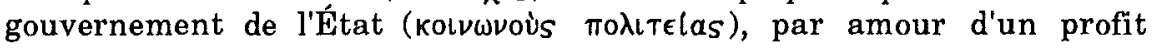
insignifiant, et s'il rompt les principaux liens de la communauté, que soit appliquée la loi suivante qui, avec l'aide des dieux, concourt à la cohésion de la cité (...).

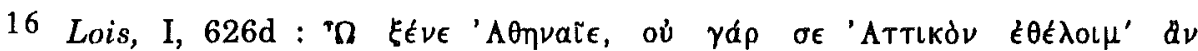

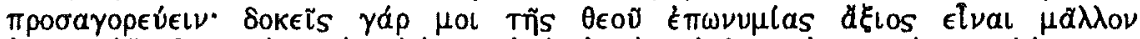

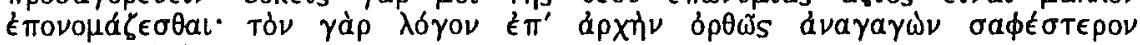
$\dot{\epsilon} \operatorname{mol} \eta \sigma a s(.$.$) .$

17 Cf. Crat, 407a-c.

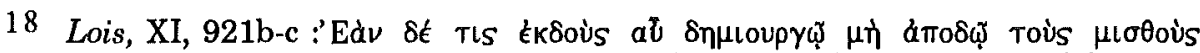

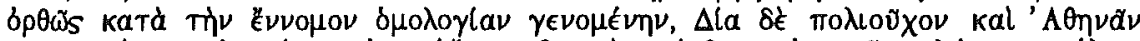

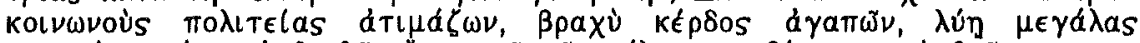

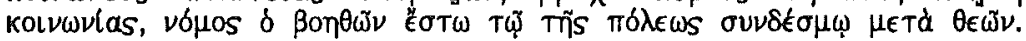


Ce n'est pas d'Athènes qu'il est question ici, mais de la cité que Platon imagine en Crète. Dans cet État idéal, Athéna et Zeus occupent une place centrale. Le devoir du législateur sera, en premier lieu, d'y délimiter un sanctuaire d'Hestia, de Zeus et d'Athéna, de l'appeler Acropole et de l'entourer d'une enceinte ${ }^{19}$. Dans chacun des bourgs occupant le centre des douze quartiers partant de l'Acropole, il devra également fonder des sanctuaires pour ces trois dieux, quelles que soient les divinités locales ${ }^{20}$.

Hestia, Zeus et Athéna sont donc les dieux prépondérants, nommément cités, que Platon établit dans le nouvel État. La présence d'Hestia se comprend aisément. Elle est en effet la déesse du commencement ${ }^{21}$, déesse immobile qui reste dans les demeures divines et humaines sans jamais les quitter ${ }^{22}$, déesse du foyer tant domestique que national, qui peut être Boudal $\alpha^{23}$, comme Zeus et Athéna. Hestia assiste tout naturellement à la fondation des cités dont elle symbolise la pérennité 24 .

Le législateur se doit également d'introduire Zeus, maître suprême des dieux et des hommes, comme le rappelle le Banquet ${ }^{25}$, ainsi qu'Athéna qui partage avec son père le gouvernement des cités. Ancienne déesse mycénienne des acropoles et des palais ${ }^{26}$, elle est restée, à Athènes du moins, la principale divinité poliade. Et si la cité décrite dans les Lois n'est pas la métropole de l'Attique, elle s'en inspire

19 Lois, V, 745b.

20 Lois, VIII, 848d.

21 Cf. l'expression «commencer par Hestia» : ARISTOKRITos, 493 F 5 Jacoby; Platon, Euthyphron, 3a; ARISTOPHANe, Guêpes, 846c, etc.

22 Cf. Platon, Phèdre, 247a; Hymne ps. -hom. à Hestia (1), 1-6; Hymne ps. -hom. à Aphrod, 21-32.

23 Cf. Eschine, Sur l'ambassade, 45.

24 Sur Hestia, cf. M.P. NILsson, Geschichte der griechischen Religion, t. I, 2e éd., Munich, 1955, p. 337-338; P.-M. SchUHL, Le joug du bien, in Mélanges Ch. Picard, Paris, 1949, p. 965 et sv.; J.-P. Vernant, Mythe et pensée chez les Grecs, Paris, 1965, p. 198-199.

25 Banquet, 197b : Selon Agathon, Éros a inspiré à Apollon le tir à l'arc, la médecine et la divination, aux Muses la musique, à Héphaistos le travail du métal, à Athéna le tissage, à Zeus le gouvernement des dieux et des hommes.

26 Cf. N. PAPACHATZis, Les origines de la déesse Athéna, in Kernos, 1 (1988), p. 79 92. 
manifestement ${ }^{27}$. C'est en partie pour cette raison qu'elle reçoit un culte au centre de la cité idéale et qu'elle y joue un rôle important, qui ne se

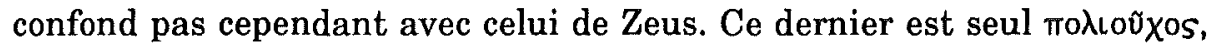
Athéna partage le gouvernement avec lui. Athéna et Zeus défendent les droits des artisans, la première parce qu'elle est particulièrement liée à cette corporation, le second parce qu'il est le gardien de l'ensemble des lois, quel que soit le groupe de citoyens concerné. Athéna et Zeus sont donc solidaires, mais leurs fonctions restent bien distinctes.

Dans l'Athènes historique, le père et sa fille sont également réunis. $\mathrm{Au}$ sophiste Dionysodore exilé à Athènes qui s'étonne que ses nouveaux concitoyens n'aient pas de Zeus matp̣̄os, Socrate explique ${ }^{28}$ :

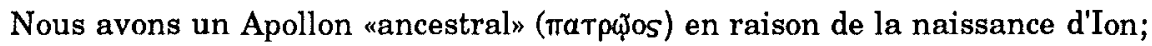
Zeus n'est pas appelé «ancestral» chez nous, mais «dieu de l'enclos» et «de la phratrie», et Athéna "déesse de la phratrie».

Répondant à la question de Dionysodore, Socrate nomme son dieu «ancestral», Apollon, et indique ensuite des épiclèses portées par Zeus chez les Athéniens. Le Zeus Ë

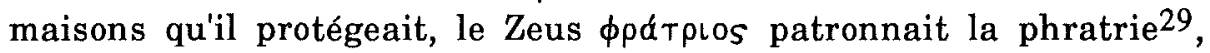
comme Athéna. Socrate introduit ainsi le nom de la principale déesse de la cité et mentionne une association typiquement athénienne, la phratrie. Apollon, Zeus et Athéna sont fréquemment associés à Athènes,

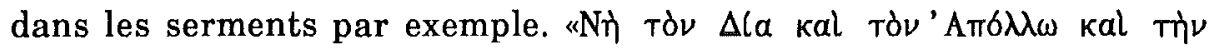

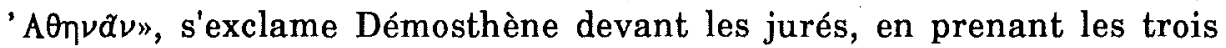
dieux à témoin de la véracité de ses dires ${ }^{30}$. Apollon, Zeus et Athéna constituent pour Socrate les éléments fondateurs, au sens propre comme

27 Cf. M. PIÉrart, Platon et la cité grecque. Théorie et réalité dans la constitution des Lois, Bruxelles, 1974, p. 464-466.

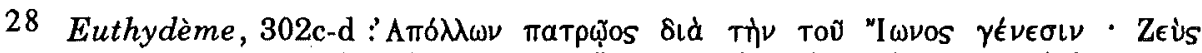

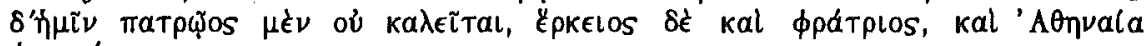
фpatpla.

29 Cf. J. Mikalson, Athenian Popular Religion, University of North Carolina Press, 1983, p. 70, 72, 83-85, 98.

30 DÉMOSTHÈne, Plaidoyers politiques, 21 (Contre Midias), 198. Sur les serments par Apollon, Zeus et Athéna, cf. A. B. Cook, Zeus, vol. II, 1, Cambridge, 1925, p. 730 . 
au sens figuré, d'Athènes. Ils sont pour lui les dieux «ancestraux et

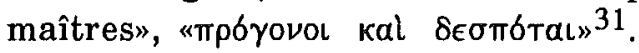

Lors de la fête des Apatouries, au cours de laquelle les nouvelles classes d'âge faisaient leur entrée dans les phratries, Athéna et Zeus $\phi \rho \alpha$ T $\iota$ o recevaient un culte particulier. Ils présidaient ainsi à l'intégration des jeunes au monde des adultes. Dans la cité des Lois, c'est dès leur première année que les enfants, garçons et filles, doivent être inscrits dans les phratries, afin que, faisant partie de ces associations patronnées par les divinités poliades, leur statut de citoyen soit désormais reconnu ${ }^{32}$.

Il est un dernier passage où Athéna et Zeus sont réunis. Dans la République, Socrate évoque l'épisode de la guerre de Troie, raconté par Homère, où Athéna, à l'instigation de Zeus, incite Pandare à lancer une flèche sur Ménélas et à rompre ainsi la paix entre Achéens et Troyens. Mais Platon condamne cette fable, et d'autres également véhiculées par les poètes, parce qu'elle attribue aux dieux un comportement immoral, indigne de leur perfection divine ${ }^{33}$.

C'est donc une déesse purifiée que le philosophe propose à ses lecteurs, à tel point qu'il se compare à elle quand elle conseille Diomède au cours du même épisode de la guerre de Troie. Athéna est un guide, non une déesse partisane. Quand elle intervient dans la vie des hommes, à titre particulier, comme pour Diomède, ou en tant que déesse poliade, c'est toujours pour leur apporter de l'aide. Athéna, première déesse d'Athènes, et Zeus, premier des Olympiens, s'associent pour assurer la prospérité de la ville au centre de laquelle ils siègent.

Athéna-Héphaistos (-Arès). Héphaistos est, avec Zeus, le dieu auquel Athéna est le plus fréquemment associée. «Entre Héphaistos et leur déesse, les Athéniens ont établi une affinité constante, attestée par le culte, par les monuments, par la poésie, mais inconnue en dehors de l'Attique. Lorsque les textes les évoquent conjointement, c'est l'un et

31 Euthydème, 302d. Il n'y a pas à proprement parler d'autre association d'Athéna et d'Apollon, ni dans le Cratyle (404b), ni dans le Banquet (197a-b). Cf. notes 25 et 35 .

32 Lois, VI, 785a-b. Cf. M. PIÉRART, op. cit. (n. 27), p. 54-56.

33 Rép., II, 379e-380a. 
l'autre comme artisans». L'œuvre de Platon confirme ce jugement de Marie Delcourt ${ }^{34}$.

Passons rapidement sur les énumérations de dieux dans le Cratyle et dans le Banquet ${ }^{35}$, et tournons-nous vers deux mythes, ceux du Protagoras et du Politique, où Athéna et Héphaistos jouent un rôle remarquable en tant que détenteurs des "T $\langle\chi \nu$ al». Dans le dialogue qui porte son nom, le sophiste Protagoras, pressé par Socrate de prouver que la vertu peut s'enseigner, entreprend de le démontrer en ayant recours, dans un premier temps, à un mythe, celui où Prométhée et Épiméthée reçoivent des dieux la mission d'attribuer aux êtres vivants des qualités appropriées. On sait comment le second oublia l'homme dans sa distribution ${ }^{36}$.

Alors Prométhée, ne sachant quel moyen de salut il pourrait trouver pour l'homme, vole la science des arts d'Héphaistos et d'Athéna en même temps que le feu.

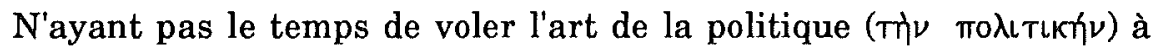
Zeus, il pénètre dans l'atelier commun d'Héphaistos et d'Athéna, atelier où ils aiment pratiquer ensemble les arts, et dérobe l'art du feu ( $\tilde{K}^{\mu} \mu \pi \nu \rho \nu$ $\tau \epsilon \chi \nu \eta \nu)$ d'Héphaistos et les autres techniques qui appartiennent à Athéna ${ }^{37}$. Selon Protagoras, cette aide ne suffira pas à sauver l'homme : Zeus devra lui faire don de la $\Delta(\kappa \eta$ et de l'Al $\delta$ w̃s pour lui permettre de se défendre contre les animaux et de survivre en communauté ${ }^{38}$.

Le même épisode de «l'histoire» humaine apparaît dans le Politique ${ }^{39}$, mais avec plusieurs modifications. L'Étranger d'Élée raconte comment, lorsque le monde abandonné par son pilote se mit à

34 M. Delcourt, Héphaistos ou la légende du magicien, Paris, 1982 (1re éd., Liège, 1957), p. 193. Notons qu'Athéna, Héphaistos et Prométhée recevaient un culte à l'Académie. Cf. L. SÉCHAN, Le mythe de Prométhée, Paris, 1951, p. 4-5.

35 Crat., 404b : Hermogène demande les étymologies des noms de Déméter, Héra, Apollon, Athéna, Héphaistos, Arès; Banquet, 197a-b : Éros a inspiré différents arts à Apollon, aux Muses, à Héphaistos, à Athéna, à Zeus.

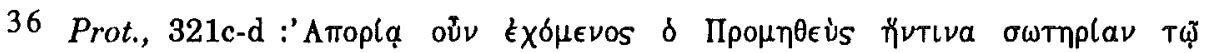

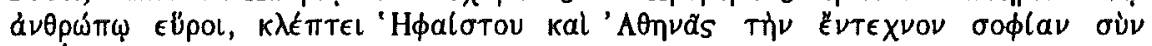
זupl.

37 Prot., 321d-e.

38 Prot., 322b-c.

39 Polit., 274b-d. 
tourner dans l'autre sens, lorsque se termina le règne de Cronos et que

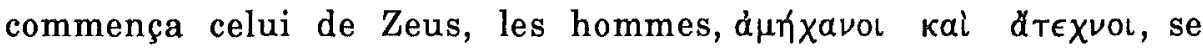
trouvèrent dans une grande détresse. Les dieux leur firent alors divers dons. Prométhée leur apporta le feu; Héphaistos et "celle qui exerce les

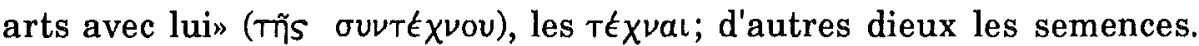
Et ils accompagnèrent ces présents de l'instruction et de l'éducation qui

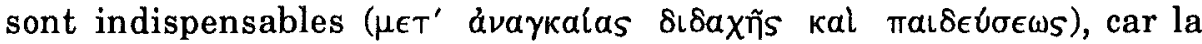
possession des arts sans la science de leur utilisation est inutile.

Un extrait des Lois peut être mis en parallèle avec ces deux mythes ${ }^{40}$. À la suite d'un déluge ne survécurent du genre humain que quelques bergers, habitants des montagnes. Point de guerre, point de politique à cette époque. En raison de leur petit nombre et des ressources que leur fournissait la nature, les hommes vivaient en parfaite harmonie, sans connaître ni pauvreté ni richesse excessives. Point de métallurgie non plus. Les métaux et l'art de les utiliser avaient disparu 41 , la paix rendait les armes inutiles; les arts "plastiques et du tissage», c'est-à-dire ceux qui leur fournissaient vêtements, couvertures, maisons et ustensiles assuraient leur bien-être ${ }^{42}$.

L'importance accordée aux arts d'Athéna, d'Héphaistos et de Zeus varie donc en fonction, non seulement de la personne qui parle et de la thèse qu'elle soutient, mais aussi de la situation humaine décrite. Protagoras défend la démocratie et montre donc Zeus distribuant la

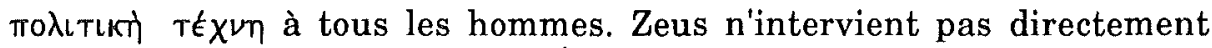
dans le récit du Politique, car l'Étranger estime qu'il lui est désormais possible de découvrir une meilleure définition du roi et du politique, et il arrête là son récit ${ }^{43}$. La République décrit une époque qui apparaît idyllique, bien qu'elle succède à un cataclysme, où la nature n'est pas hostile aux hommes et où ceux-ci, par conséquent, vivent dans la paix et la vertu ${ }^{44}$. La fonction technique représentée par Athéna, généralement accompagnée d'Héphaistos et parfois de Prométhée, est donc toujours présente dans le mythe ${ }^{45}$.

\footnotetext{
40 Lois, III, 678e-679d.

41 Lois, III, 678c-e.

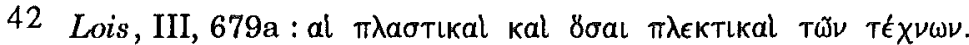

43 Polit., 274e.

44 Lois, III, 679c.

45 Cf. J. -P. VeRnANT, op. cit. (n. 24), p. 264 et n. 60 p. 286.
} 
Dans la cité des Lois, la classe des artisans «qui, par les techniques nous ont aidés à nous procurer ce mode de vie» est consacrée à Hephaistos et à Athéna, tandis que cette dernière, associée à Arès, protège les guerriers, gardiens des ouvrages des artisans ${ }^{46}$. Athéna et Héphaistos sont de nouveau présentés comme les dieux artisans, mais Athéna, contrairement à son frère, ne se limite pas à cette fonction. Alors que dans la mythologie grecque, Athéna et Arès sont le plus souvent opposés, et que la déesse de l'intelligence l'emporte sur le dieu de la violence, Platon les associe. Arès, dieu tout à fait secondaire dans le corpus platonicien, perd son caractère violent et irréfléchi, pour jouer avec l'aide d'Athéna le rôle positif de protecteur de la cité.

De même qu'Athéna et Zeus veillent au respect des lois, de même qu'Athéna et Héphaistos protègent les artisans, de même Athéna et Arès assurent-ils la sécurité des artisans, et des citoyens de façon générale. Athéna est donc partout présente. Elle sert en effet de trait d'union entre les dieux. Fille de Zeus, elle attire sur les hommes la bienveillance et les qualités de souverain de son père; déesse technicienne, comme Héphaistos, mais douée d'une intelligence hors du commun, elle est en quelque sorte maître d'œuvre là où Héphaistos n'est qu'artisan 47 ; déesse de la guerre, elle la mène intelligemment, alors qu'Arès ne connaît que le combat destructeur. Chez Platon, c'est grâce à Athéna que les qualités de chaque dieu sont le mieux mises en relation pour assurer de façon efficace le bon fonctionnement des cités.

Une autre cité, qui n'est ni la cité idéale des Lois, ni la cité universelle des hommes, jouit de la présence d'Athéna et d'Héphaistos, c'est l'Athènes primitive qui remporta la victoire contre l'Atlantide et qu'évoque le mythe du Critias. Quand les dieux se partagèrent le monde,

Héphaistos et Athéna, qui ont la même nature, à la fois parce qu'ils sont frère et sœur issus du même père et parce que leur amour de la science et des techniques les pousse vers les mêmes buts, reçurent ainsi à eux deux un seul lot, cette contrée, car elle était naturellement apparentée et adaptée à la vertu et à la

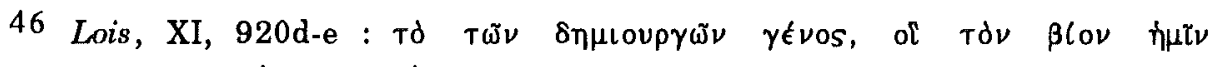

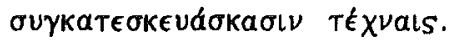

47 Cf. M. DetIENNE- J. -P. VERNANT, Les ruses de l'intelligence, Paris, 1974, p. 175 et n. 49 p. 185. 
sagesse; ils y firent naître des gens de bien comme autochtones et organisèrent la ville selon leur volontét8.

C'est donc en raison de leur parenté et de leurs goûts communs qu'Athéna et Héphaistos agissent de concert. Ils ne font qu'un, l'un avec l'autre et tous les deux avec la terre qu'ils ont reçue et le peuple qu'ils y ont fait naître. Leur union fait la force de la ville. Ensemble, ils l'ont

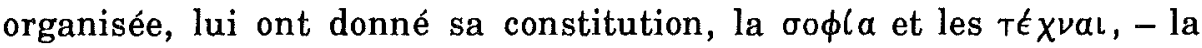
science et les techniques qui sont de nouveau réunies. La $\phi \rho b v \eta \sigma s$ innée $^{-}$ du peuple athénien ${ }^{49}$ caractérise également la déesse ${ }^{50}$, et même Héphaistos qui, par son association avec Athéna, devient un dieu sage et protecteur de la cité.

La disposition du temple et des habitants révèle la même symbiose. Le sommet de l'Acropole des temps anciens, alors vaste colline fertile, était occupé en son centre par le sanctuaire d'Héphaistos et d'Athéna. Autour du temple étaient établis les guerriers, et aux abords de l'Acropole les artisans et les paysans ${ }^{51}$.

Cette disposition rappelle les trois classes de la République 52 composées des artisans et des paysans, des guerriers et des philosophes. Le statut d'Athéna et d'Héphaistos n'est donc pas exempt d'une certaine ambiguïté. Car ils sont en même temps les maîtres d'Athènes et les patrons de la troisième classe. La même ambiguïté marque le statut des artisans. On pourrait s'attendre, puisqu'ils sont protégés par les dieux de la cité, à ce qu'ils jouissent d'un statut privilégié. Or ils occupent le bas de hiérarchie sociale. L'explication de cette ambiguïté réside sans doute dans le fait que, pour Platon, chaque TÉ $\chi \chi^{\nu \eta}$ représente un domaine de compétence défini. Les artisans possèdent donc un savoir clairement délimité qui peut servir de modèle pour la définition de notions plus

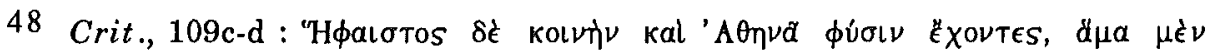

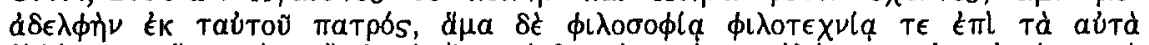

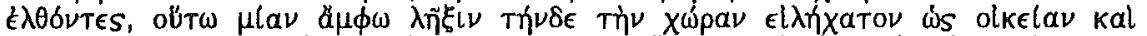

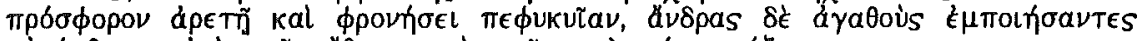

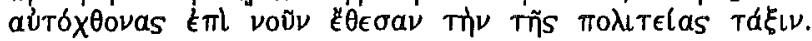

49 Crit., 109d.

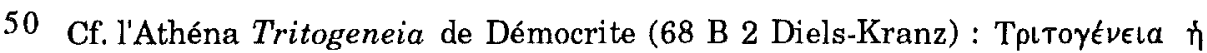
'A

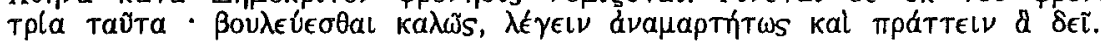

51 Critias, 111e-112e.

52 Rép., III, 415a. 
complexes ${ }^{53}$. Mais s'ils détiennent une $\tau \in ́ \chi \chi \eta \eta$ précise, ils sont dépourvus de la science suprême des philosophes. C'est cette ooфla que Platon recherche et qui doit guider la $T \hat{\epsilon} \chi \nu \eta$ des artisans ${ }^{54}$. Ce n'est donc que sous l'autorité des philosophes que les artisans peuvent contribuer à faire naître une cité aussi remarquable que l'Athènes primitive.

Athéna-Neith. Et Athéna-Poséidon? Dans le Timée, Critias révèle à Socrate le récit que son grand-père a entendu de la bouche de Solon. Ce dernier, «le plus sage des sept sages» 55 , a recueilli en Égypte des informations sur l'ancienne cité d'Athènes et sur l'exploit admirable entre tous qu'elle accomplit ${ }^{56}$. Ainsi commence la narration :

(...) pour les habitants, c'est une certaine divinité qui a fondé la ville (Saìs). En égyptien, son nom est Neith et en grec, à ce qu'ils disent, Athéna 57 .

Cette interpretatio Graeca ne devait pas surprendre les lecteurs de Platon. Neith est généralement assimilée par les Grecs à Athéna, qui, comme elle, est liée à la guerre et au tissage et est honorée pour sa sagesse. La ville de Saïs passait même pour être une colonie d'Athènes ${ }^{58}$.

Le prêtre égyptien que Solon a rencontré explique ensuite qu'Athéna et Neith ne sont en fait qu'une même déesse qui a fondé Athènes mille ans avant Saïs, il y a de cela neuf millénaires, et a donné à chaque ville sa constitution 59 . Les Grecs en ont perdu le souvenir en raison des

53 Cf. G. Cambiano, Platone e le tecniche, Turin, 1971, p. 80-89.

54 Cf. A. Moтte, Pour l'authenticité du Premier Alcibiade, in AC, 30 (1961), p. 532.

55 Timée, 20e, 21b; cf. Prot., 343a.

56 Dans aucune ouvre ou fragment conservés, Solon ne parle de l'Égypte, et aucun témoignage antérieur à Platon ne concerne l'Atlantide. Solon est plusieurs fois mentionné par Platon en tant que sage, poète ou législateur exceptionnel (Charmide, 155a; Lachès, 188b, 189a; Banquet, 209d; Phèdre, 278c, etc. ). On peut donc considérer que c'est Platon qui parle par sa bouche.

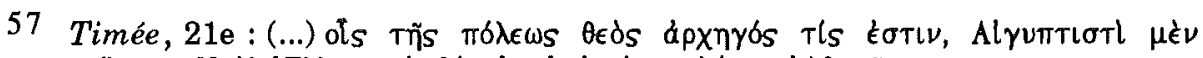

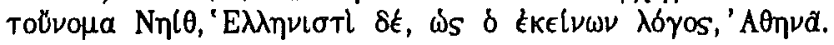

58 Cf. A. RusH, art. Neith, in RE, XVI, 2 (1935), col. 2205-2206; H. BONNET, Reallexicon der Ägyptischen Religionsgeschichte, Berlin, 1952, p. 517; J. Quaegebeur - W. Clarysse - B. VAN MAEle, Athêna, Nêith and Thoêris in Greek Documents, in ZPE, 60 (1985), p. 217-232.

59 Timée, 23d-24e. Cf. Critias, 113a : Solon a rendu leur appellation grecque aux dieux dont le nom a été transposé par les Égyptiens dans leur langue. 
cataclysmes successifs qui ont détruit leur civilisation, mais les inscriptions des temples égyptiens en gardent la mémoire ${ }^{60}$.

L'Athènes primitive était une ville parfaite réalisant la cité idéale décrite dans la République 61 . Terre jouissant d'un climat apte à porter les hommes les plus intelligents ${ }^{62}$, elle a été choisie par Athéna qui a

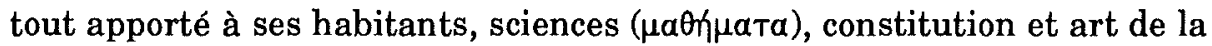
guerre qu'elle a elle-même enseigné ${ }^{63}$. Si une importance particulière est accordée à l'aspect guerrier de la déesse, alors qu'il est laissé au second plan dans les autres dialogues, c'est parce que le principal exploit de la cité est sa victoire dans la lutte contre l'Atlantide. L'Athéna du Timée et du Critias est une déesse qui «aime à la fois la guerre et la

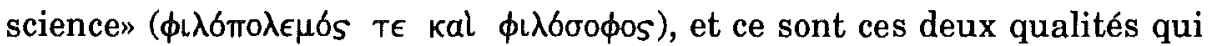
lui permettent de créer une cité invaincue au combat et dotée des meilleures lois ${ }^{64}$.

Une place y est-elle accordée aux deux divinités qui, dans la religion grecque, sont avec Athéna les principaux acteurs de la naissance d'Athènes, Héphaistos et Poséidon?

Après avoir indiqué qu'Héphaistos partage avec Athéna la ville et le temple qui en occupe le centre, Platon rappelle que le dieu est, avec Gè, l'ancêtre des Athéniens. Mais aucune précision n'est donnée sur le rôle qu'il joue dans l'organisation de la cité. L'épisode scabreux d'Héphaistos poursuivant Athéna et laissant tomber sa semence sur le sol n'est pas rappelé. Il n' y a aucune mention non plus de la lutte entre Athéna et Poséidon pour la possession de l'Attique. Les dieux de Platon se partagent paisiblement le monde ${ }^{65}$. Athéna et Poséidon ne sont pas pour autant associés, comme Athéna et Arès. Leur rivalité est bien présente, mais elle a été transposée sur le plan d'une guerre entre deux États divins, Athènes et l'Atlantide.

Ces deux pays se ressemblent à bien des égards. La terre y fait pousser ses fruits en abondance, l'eau y est intarissable, le territoire

\section{Timée, 23a-b.}

61 Cf. le début du Timée (19b-c).

62 Timée, 24c.

63 Timée, 24a-c.

64 Timée, 23c-d.

65 Critias, 109a-b. 
savamment organisé ${ }^{66}$. Pourtant, si on les examine plus attentivement, on constate que l'un est l'envers de l'autre. Sur l'île de Poséidon, sont construits un grand nombre de temples destinés à de nombreux dieux; à Athènes, un temple unique d'Athéna et d'Héphaistos s'élève sur l'Acropole ${ }^{67}$. Le temple de Poséidon, bariolé d'or, d'argent, d'orichalque, a une apparence barbare; Athènes n'utilise pas ou peu les métaux ${ }^{68}$. L'île possède de nombreux ports et un commerce important; l'économie d'Athènes repose essentiellement sur l'agriculture ${ }^{69}$. Alors qu'elle a toutes les richesses à sa disposition, l'Atlantide développe des ambitions impérialistes, et, dans son invasion de l'Europe et de l'Asie, menace la cité d'Athéna ${ }^{70}$.

La race issue de Poséidon connaît une dégénérescence de plus en plus marquée au fur et à mesure que sa nature divine, mélangée à des éléments humains, s'estompe. Excès divers, impérialisme, déclin de la race : l'Atlantide était perdue d'avance dans sa lutte contre Athènes. Zeus lui-même veut la punir. L'île sombre dans les flots, victime d'une double défaite, contre Athènes et vis-à-vis d'elle-même.

Athènes et l'Atlantide sont des États jumeaux, parfaits en apparence, mais chez l'un cette perfection est réelle, tandis que chez l'autre elle n'est qu'illusion. L'île présente de nombreux aspects, condamnés par Platon, de l'Athènes classique ${ }^{71}$. L'Athènes des premiers temps, au contraire, doit sa supériorité à deux qualités, la juste mesure et la cohésion. Elle ignore les excès dans l'utilisation des richesses, dans le commerce, dans l'architecture. Les différents groupes de citoyens, sans être égaux, s'acquittent de la tâche dont ils sont chargés, assurant ainsi la prospérité et l'équilibre de l'ensemble de la ville.

Athènes ne survit pourtant pas à sa rivale. Un tremblement de terre et un déluge l'engloutissent le jour même. Poséidon se venge-t-il ? Il semble plutôt qu'Athènes ne puisse demeurer une fois l'Atlantide disparue, car les deux États se contrebalançaient. La défaite de l'Atlantide met fin à cet âge des cités divines, peuplées d'hommes faits à

66 Critias, 110d-e, 111b-d, 112c-d, 113e, 114e-115b.

67 Critias, 112b, $117 \mathrm{~d}$.

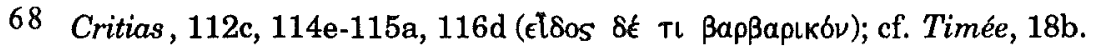

69 Critias, 110e-111a, 117d. Cf. M. PiéraRT, op. cit. (n. 27), p. 48.

70 Timée, $24 \mathrm{e}$.

71 Cf. P. LÉVÊque - P. VIDAL-NAQUET, Clisthène l'Athénien, Paris, 1964, p. 134-140. 
l'image des dieux ${ }^{72}$. De même que l'âge d'or hésiodique est suivi de périodes moins brillantes, de même la défaite, inévitable, de l'île de Poséidon marque-t-elle la fin d'une période et l'entrée dans un monde moins proche des dieux, moins bon, plus humain. De ces temps anciens, il ne reste qu'une ville en Égypte, Saïs, qui, parce qu'elle a été fondée elle aussi par la déesse, mais en second lieu ${ }^{73}$, parce que ses pierres gardent le souvenir du passé et que son peuple a conservé certaines institutions anciennes ${ }^{74}$, permet d'imaginer la splendeur de l'Athènes primitive.

Cette brève revue appelle plusieurs conclusions. Relevons tout d'abord que l'Athéna de Platon gouverne la cité-État et patronne les $T \epsilon \chi \chi v a$. Sous ces aspects, elle n'est pas différente de la déesse protectrice d'Athènes. Les fêtes illustrent bien, à Athènes comme chez Platon, le lien étroit qui existe entre la déesse et la cité. C'est à l'occasion des récitations de poèmes qui avaient lieu lors des Apatouries que la conversation a été amenée sur Solon et que Critias l'ancien a répété aux jeunes qui l'entouraient le récit qu'il avait entendu du sage ${ }^{75}$, et c'est lors des Panathénées que Critias le jeune fit à son tour le récit à Socrate ${ }^{76}$. Les mêmes fêtes permirent aux philosophes du Parménide de mener une longue conversation 77 . Les concours de rhapsodie des Panathénées sont mentionnés deux fois par Platon, dans l'Ion où Socrate souhaite à son interlocuteur de remporter un même succès aux fêtes d'Athènes qu'à celles d'Épidaure ${ }^{78}$, et dans l'Hipparque où il est rappelé que c'est le fils de Périclès qui obligea les rhapsodes à réciter les poèmes homériques aux Panathénées ${ }^{79}$. Ailleurs, Socrate récuse les

72 Timée, $24 \mathrm{~d}$.

73 Il est dit deux fois que les Athéniens furent les premiers à recevoir les enseignements de la déesse : Timée, $24 \mathrm{~b}$ et c.

74 Timée, 24a-b.

75 Timée, $21 \mathrm{~b}$.

76 Timée, 21a, 26e.

77 Parm., 127a.

78 Ion, $530 \mathrm{~b}$.

79 Hipparque, 228b. 
querelles auxquelles la tradition prétend que les dieux se sont livrés, querelles représentées notamment sur le péplos offert à Athéna ${ }^{80}$.

Les Panathénées reflètent l'unité politique des citoyens athéniens et l'éclat de leur cité. Rencontre des générations - rencontre de Critias au seuil de ses quatre-vingt-dix ans avec Critias le jeune alors âgé d'environ dix ans ${ }^{81}$-, discussions entre philosophes athéniens et étrangers, concours divers (rhapsodie, pyrrhique), la cité célèbre, dans une effervescence politique, religieuse et intellectuelle, la déesse qui la protège et patronne les multiples aspects de la vie en société.

La figure d'Athéna telle que la montre Platon n'est pas dépourvue cependant de traits originaux. La première loi que devra respecter toute personne parlant des dieux dans la République est que « la divinité n'est pas responsable de tout, mais seulement du bien» ${ }^{82}$. Il sera donc interdit de raconter des fables montrant les dieux nuisant aux hommes. Platon cite de nombreux exemples, dont celui de Zeus et d'Athéna trompant Pandare. Athéna, comme tous les dieux de Platon, agira donc toujours d'une manière conforme à la morale.

Platon n'exploite pas pour autant sur le plan philosophique la déesse ainsi purifiée des scories de la mythologie, comme il le fait avec Zeus, Éros ou Apollon. Athéna appartient à la cité de Platon plus qu'à son système philosophique. Elle est uniquement une déesse de la cité et des arts, et ce rôle n'est nullement négligeable, car l'élaboration d'un État idéal est une des préoccupations majeures de Platon et que les arts y sont toujours présents. Si l'on s'interroge sur les $\tau \dot{E} \chi \nu$ a auxquelles Athéna préside, on trouvera peu d'indications dans les dialogues. C'est l'ensemble de la fonction technique qu'elle représente avec Héphaistos, et non tel art en particulier. On peut également remarquer que Platon accole peu d'épiclèses au nom d'Athéna ${ }^{83}$. Elle est $l a$ déesse, í $\theta \epsilon \delta s, d u$

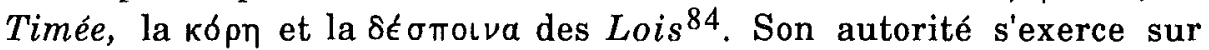
l'ensemble de la ville et des arts plutôt que dans un domaine étroitement délimité.

80 Euthyphron, 6b-c.

81 Timée, 21a.

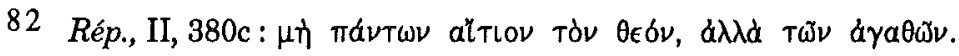

83 Elle est uniquement $\phi \rho a \tau p l a$.

84 Timée, 21a, 23d; Lois, VII, $796 \mathrm{~b}$. 
Athéna sert de trait d'union entre Zeus, Héphaistos et Arès. Elle représente la juste mesure dans les rapports entre dieux et assure la sage organisation de la cité. Athéna doit ce rôle à deux facteurs. Fille préférée de Zeus, elle est l'intermédiaire privilégiée entre son père et les autres Olympiens et entre ce dernier et les hommes. D'autre part, elle est l'Intelligence, le Cratyle le souligne, et cette qualité lui permet d'être la juste modératrice et conseillère dans l'organisation divine et humaine des cités. Son intelligence appliquée aux différentes situations de la vie en société rend chacune de ses interventions efficace : quand elle aide Diomède-Alcibiade, quand elle patronne les artisans ou quand elle organise un État et le conduit à la victoire.

Ainsi qu'on l'a remarqué, Athéna apparaît beaucoup plus souvent associée à d'autres dieux qu'elle n'est seule. Et, exception faite des étymologies données dans le Cratyle et du passage des Lois où elle invente une danse armée, c'est toujours lorsqu'elle collabore avec d'autres dieux qu'elle joue un rôle important - prendre part au gouvernement, protéger les artisans, etc.

La question se pose évidemment de savoir s'il en va de même avec les autres divinités. Seule une étude consacrée à l'ensemble des dieux dans l'œuvre de Platon pourrait y répondre. Pourtant, cette "sociabilité" d'Athéna ne surprend guère. La cité est un ensemble d'individus et d'institutions qui ont besoin d'être coordonnés suivant une sage organisation. Quant aux $\tau \epsilon \chi v a L$, elles ne sont vraiment utiles que si elles sont employées ensemble. L'art du gouvernement appartient à une élite formant un groupe homogène, de même que l'art des guerriers. Les artisans, au contraire, pratiquent une multitude de métiers qui, par leur réunion, fournissent au groupe social ses moyens de subsistance. L'unité de la classe des artisans est aussi nécessaire et difficile que celle de l'ensemble de la cité. Toutes deux ne sont possibles que sous l'égide de la sage Athéna.

Si Athéna occupe, dans les dialogues, une place secondaire par rapport à un Zeus, un Apollon voire un Éros, elle n'est pas pour autant une figure sans importance. Car elle est la déesse d'Athènes, et l'Athènes de Platon n'est pas seulement la métropole de l'Attique, mais aussi la cité idéale qu'il voudrait construire, une cité qui verrait à la fois l'organisation politique, la technique guerrière et les arts patronnés 
par l'Intelligence ${ }^{85}$. Il est remarquable à ce propos qu'Athéna soit présente dans les quatre œuvres où Platon décrit un État, la République, le Timée, le Critias et les Lois ${ }^{86}$. Il n'y a donc pas de cité platonicienne sans Athéna. Ou bien elle partage le gouvernement avec Zeus, ou bien elle assure elle-même la souveraineté, ou bien elle détient avec Héphaistos les arts qui fournissent le $\beta$ lov aux hommes. De même que l'Athènes de Platon est une ville exemplaire, parfois bien éloignée de la réalité, de même l'Athéna de Platon est-elle une déesse purifiée et idéalisée, qui n'existe pas indépendamment de la cité, mais sans laquelle il n'est pas de cité parfaite.

Rue des Français, 19

Catherine LECOMTE

B - 4430 ANS

85 Supériorité à la guerre, amour des arts et intelligence sont à la fois les qualités d'Athéna et celles que Périclès attribue aux Athéniens dans son discours funèbre (Thuc., II, 35 et sv. ). Cf. C.J. Herington, Athena Parthenos and Athena Polias, Manchester, 1955, p. 56.

86 Ce qui n'est pas le cas de tous les dieux, par exemple de Poséidon et de Dionysos (cf. P. VICAIRE, Platon et Dionysos, in BAGB, 4e série, $\mathrm{n}^{\circ} 3$, oct. 1958, p. 15-26). 